\title{
ORIGINAL ARTICLE \\ Decrease in abundance of bacteria of the genus Bifidobacterium in gut microbiota may be related to pre-eclampsia progression in women from East China
}

\author{
Tingting Miao ${ }^{1,2 \#}$,Yun Yü, Jin Sun², Aiguo Ma², Jinran Yư ${ }^{4}$, Mengjun Cui ${ }^{5}$, Liping Yang ${ }^{6}$ \\ and Huiyan Wang ${ }^{6 *}$
}

\begin{abstract}
'Department of Education, Changzhou Maternity and Child Health Care Hospital Affiliated to Nanjing Medical University, Changzhou, China; ${ }^{2}$ Institute of Nutrition and Health, Qingdao University, Qingdao, China; ${ }^{3}$ Department of Clinical Laboratory, Changzhou Maternity and Child Health Care Hospital Affiliated to Nanjing Medical University, Changzhou, China; ${ }^{4}$ School of Public Health, Qingdao University, Qingdao, China; ${ }^{5}$ School of Food Science and Technology, Jiangnan University, Wuxi, China; ${ }^{6}$ Department of Obstetrics and Gynecology, Changzhou Maternity and Child Health Care Hospital Affiliated to Nanjing Medical University, Changzhou, China
\end{abstract}

\section{Popular scientific summary}

- Pre-eclampsia is a common hypertensive disorder during pregnancy which may be related to the alternaions of gut microbiota, however investigations on gut microbiota in pre-eclampsia are rare.

- Decreased in abundance of bacteria of the genus Bifidobacterium showed a negative correlation with the systolic blood pressure, diastolic blood pressure, and dyslipidemia.

- Our data could help to monitor the health of pregnant women and may be helpful for preventing and assisting treatment of pre-eclampsia by increasing dietary fiber or probiotics supplement.

\section{Abstract}

Background: Pre-eclampsia (PE) can result in severe damage to maternal and fetal health. It has been reported that gut microbiota $(\mathrm{GM})$ had important roles in regulating the metabolic and inflammatory responses of the mother. However, investigations on GM in PE are rare.

Objective: The objective of the present study was to investigate the changes of GM in PE and how to alter the GM composition in PE by dietary or dietary supplements.

Design: We analyzed the composition changes in GM as well as the relationship between bacteria of different genera and clinical indices by amplifying the V4 region of the $16 \mathrm{~S}$ ribosomal RNA gene in 12 PE patients and eight healthy pregnant women in East China.

Results: In the PE group, the Observed Species Index was lower than that in the control group, indicating that the $\alpha$-diversity of the microbiome in the PE group decreased. At phylum, family, and genus levels, the relative abundance of different bacteria in PE patients displayed substantial differences to those from healthy women. We noted a decreased abundance of bacteria of the phylum Actinobacteria $(P=0.042)$, decreased abundance of bacteria of the family Bifidobacteriaceae $(P=0.039)$, increased abundance of bacteria of the genus Blautia $(P=0.026)$ and Ruminococcus $(P=0.048)$, and decreased abundance of bacteria of the genus Bifidobacterium $(P=0.038)$. Among three enriched genera, bacteria of the genus Bifidobacterium showed a negative correlation with the systolic blood pressure (SBP), diastolic blood pressure (DBP), and dyslipidemia, which involved glucose metabolism, lipid metabolism, and the oxidative-phosphorylation pathway. The increased abundance of bacteria of the genera Blautia and Ruminococcus was positively correlated with obesity and dyslipidemia, which involved lipid metabolism, glycosyltransferases, biotin metabolism, and the oxidative-phosphorylation pathways. Moreover, women in the PE group ate more than women in the control group, so fetuses were more prone to overnutrition in the PE group.

Conclusion: There is a potential for GM dysbiosis in PE patients, and they could be prone to suffer from metabolic syndrome. We speculate that alterations in the abundance of bacteria of certain genera (e.g. increased

\#These authors contributed equally to this work. 
abundance of Blautia and Ruminococcus, and decreased abundance of Bifidobacterium) were associated with PE development to some degree. Our data could help to monitor the health of pregnant women and may be helpful for preventing and assisting treatment of PE by increasing dietary fiber or probiotics supplement.

Keywords: gut microbiota; Blautia; Ruminococcus; Bifidobacterium; pre-eclampsia

To access the supplementary material, please visit the article landing page

Received: 8 November 202I; Revised: I 5 January 202I; Accepted: 2 February 2021; Published: 28 June 202 I

$\mathrm{P}$ re-eclampsia $(\mathrm{PE})$ is a common hypertensive disorder during pregnancy. It affects an estimated 3-5\% of pregnancies worldwide and contributes substantially to the morbidity and mortality of the mother and fetus (1). One characteristic of PE is the development of concurrent hypertension and proteinuria or end-organ damage in middle- or late-stage pregnancy (2). Various angiogenic, genetic, structural, and metabolic pathways have been considered to be related to PE development, including remodeling of spiral arteries, placental oxygenation, and immune tolerance $(2,3)$. Endothelial dysfunction as well as an imbalance of angiogenic and antiangiogenic factors during pregnancy is associated with $\mathrm{PE}(2,4)$.

Gut microbiota (GM) consist of a complex and diverse community of microorganisms (5). In health, human GM interact and balance in abundance with each other to form a stable ecosystem. The latter plays a unique part in metabolism, immunity, and nutrition absorption (6). There are $\sim 1,014$ known bacteria in the intestine, including $>2,000$ species and 12 phyla (7). GM can produce diverse compounds that can regulate the activities of distal organs. These actions could facilitate insulin resistance by inducing chronic inflammation, which has an important role in disease development (6). The various factors that affect intestinal microecology, such as consumption of a high-fat diet, obesity, and an excessive increase in the gestation period, are independent risk factors for cardiovascular disease and metabolic disease. Otherwise, these factors can induce metabolic abnormalities, excessive oxidative stress, and immunotolerance imbalance by changing intestinal microecology and alterations in abundance of specific bacteria, which can result in the occurrence and development of PE.

However, the relationship between the diversity of intestinal microflora and $\mathrm{PE}$ in women from East China is not known (although some results are available for women in South China [8]). Here, we analyzed GM differences between women with PE and healthy pregnant women (control group). We also assessed the relationship between the abundance of different GM and clinical indices. In this way, we offered new concepts on the prediction of $\mathrm{PE}$ and potential interventions against $\mathrm{PE}$.

\section{Materials and methods}

\section{Ethical approval of the study protocol}

The study protocol was approved by the Internal Ethics Committee of the Institute of Chinese Medical Sciences, and Ethics Committee of Changzhou Maternity and Child Health Care Hospital (QNRC2016302) in Changzhou, China. The study was carried out in accordance with the Declaration of Helsinki 1964 and its later amendments. Patients provided written informed consent for collection of their stool samples for experimentation.

\section{Patients}

Patients were treated in the Department of Obstetrics and Gynecology in Changzhou Maternity and Child Health Care Hospital (which is affiliated to Nanjing Medical University) from October 2017 to April 2018. According to the current guidelines from American College of Obstetricians and Gynecologists (ACOG) (2019), the diagnostic criteria for PE were as follows: 1) systolic blood pressure (SBP) $\geq 140 \mathrm{mmHg}$ and/or diastolic blood pressure (DBP) $\geq 90 \mathrm{mmHg}$ on two occasions at least $4 \mathrm{~h}$ apart after 20 weeks of gestation in a woman with a previously normal blood pressure; 2) proteinuria $>300 \mathrm{mg} / 24$, or protein/creatinine ratio $\geq 0.3 \mathrm{mg} / \mathrm{dL}$, or dipstick reading of $2+$ (used only if other quantitative methods not available); 3) in the absence of proteinuria, new-onset hypertension with the new onset of any of the following: a) thrombocytopenia: platelet count $<100,000 \times 109 / \mathrm{L}$; b) renal insufficiency: serum creatinine concentrations $>1.1 \mathrm{mg} / \mathrm{dL}$ or a doubling of the upper limit in the absence of other renal disease; c) impaired liver function: elevated blood concentrations of liver transaminases to twice normal concentration; d) pulmonary edema; e) new-onset headache unresponsive to medication and not accounted for by alternative diagnoses or visual symptoms $(2,9)$. The age of pregnant women, gestational weeks when the fecal samples were collected, and gravidity and parity were matched in the two groups (10). None of the women in either group had: 1) gestational diabetes mellitus, hyperlipidemia, or other complications of pregnancy; 2) an infection or used antibiotics in the month before stool collection. 


\section{Sample collection and microbiome sequencing}

Feces (>250 mg) was collected using a sterilized sample box and stored at $-80^{\circ} \mathrm{C}$ within $2 \mathrm{~h}$. Afterward, the collected samples were dissolved in peptone buffer to obtain $20 \%$ bacterial solutions. Tween $20(0.5 \%)$ was added to the bacterial solutions, which were then centrifuged at $1,000 \times g$ for $5 \mathrm{~min}$ at room temperature to collect supernatants and then for an additional $5 \mathrm{~min}$ at $10,000 \times \mathrm{g}$ to collect precipitates. The latter were washed with peptone buffer, dissolved in $50 \%$ glycerol, and stored at $-80^{\circ} \mathrm{C}$ for later use.

Afterward, all the bacterial samples were centrifuged $\left(9,000 \mathrm{rpm}, 4^{\circ} \mathrm{C}\right)$ for $8 \mathrm{~min}$ before addition of $0.3 \mathrm{~g}(0.15$ $\mathrm{mm})$ and $0.1 \mathrm{~g}(0.7 \mathrm{~mm})$ of zirconia/silica beads (Biospec Products, Bartlesville, OK, USA) and $1 \mathrm{~mL}$ of lysis buffer. The Multifunctional Tissue Homogenizer $(6,300 \times g, 35 \mathrm{~s}$ of bead beating, $45 \mathrm{~s}$ on ice; $6,300 \times g$, $35 \mathrm{~s}$ of bead beating, $45 \mathrm{~s}$ on ice; Bertin Technologies, Aix-en-Provence, France) was used to homogenize samples, which were then centrifuged for $5 \mathrm{~min}\left(15,000 \times g\right.$ at $\left.4^{\circ} \mathrm{C}\right)$. Then, the supernatants were transferred to 2-mL Eppendorf (Hamburg, Germany) tubes, whereas $300 \mu \mathrm{L}$ of lysis buffer was added to the precipitates. Then, the steps mentioned above were repeated to enable combination of the supernatants into 2-mL Eppendorf tubes. Ammonium acetate $(300 \mu \mathrm{L})$ was mixed with the supernatants, which were centrifuged for $10 \min \left(15,000 \times g\right.$ at $\left.4^{\circ} \mathrm{C}\right)$. DNA was precipitated by addition of 1 volume of isopropanol, followed by centrifugation for $15 \mathrm{~min}\left(15,000 \times g\right.$ at $\left.4^{\circ} \mathrm{C}\right)$. Afterward, the precipitates were washed with $1 \mathrm{~mL}$ of $75 \% \mathrm{EtOH}$, centrifuged $\left(5 \mathrm{~min}, 15,000 \times \mathrm{g}, 4^{\circ} \mathrm{C}\right)$, dried $(15 \mathrm{~min}$, no heat, 'Auto Run' setting), and then dissolved in $100 \mu \mathrm{L}$ of

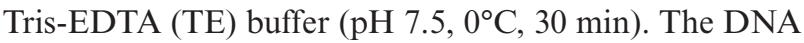
solutions were reacted with RNase A $(10 \mathrm{mg} / \mathrm{mL}$; Sangon Biotech, Shanghai, China) before purification using an Ezup $^{\circledR}$ Column Bacterial Genomic DNA Extraction Kit (Sangon Biotech) and elution in CE buffer (a buffer for DNA dissolution, $50 \mu \mathrm{L}$ ), followed by storage at $-20^{\circ} \mathrm{C}$.

Frozen samples were sequenced to amplify the V4 region of $16 \mathrm{~S}$ ribosomal RNA. Sequencing was achieved using a standard protocol for real-time reverse transcription-quantitative polymerase chain reaction employing a universal bacterial forward primer 515F (5'-GTGCCAG CMGCCGCGGTAAN-3') and reverse primer 806R (5'-GGACTACHVGGGTW TCTAAT-3'). Samples in which amplification was successful were provided by BGI (Beijing, China) as the positive control and sterile deionized water served as the negative control. Raw data were filtered to delete low-quality sequences, and then, pairedend reads were added to tags by the Fast Length Adjustment of Short reads program (FLASH, v1.2.11) to get the tags (11). The tags were clustered into OTUs with a cutoff value of 97\% using UPARSE software (v7.0.1090) (12), and chimera sequences were compared with the Gold database using UCHIME (v4.2.40) (13) to detect. Then, OTU representative sequences were taxonomically classified using Ribosomal Database Project (RDP) Classifier v.2.2 with a minimum confidence threshold of 0.6 and trained on the Greengenes database v201305 by QIIME v1.8.0 (14). The USEARCH global (15) was used to compare all Tags back to OTU to get the OTU abundance statistics table of each sample. The number of operational taxonomic units (OTUs) can be used to show the abundance of a particular species (16).

Phylogenetic Investigation of Communities by Reconstruction of Unobserved States (PICRUSt; http://picrust. github.io/picrust) was used to obtain three levels in the metabolic pathway and to ascertain the abundance table of each level (17). PICRUSt stores the orthology information from the Kyoto Encyclopedia of Genes and Genomes (KEGG) database corresponding to the Greengene ID, which can be used to obtain the Kegg Orthology (KO) corresponding to the OTUs for each sample (18). The data-library information from the KEGG database and OTU abundance were used to calculate the abundance of each functional category.

\section{Statistical analyses}

Differences in bacterial abundance between groups were observed through partial least-squares discrimination analysis (PLS-DA) based on the OTU. The relative abundance of OTUs was analyzed using the linear discriminant analysis effect size (LEfSE) algorithm to identify taxa which could display significant differences in the two groups. The $\alpha$-diversity of GM from each pregnant woman was assessed by the Simpson Index, Shannon Index, Sobs Index, and Chao Index. Significant differences between the two groups were analyzed by the Mann-Whitney $U$-test for non-parametric data. The relative abundance of GM was calculated at phylum, family, and genus levels between the two groups. HemI 1.0 was used to create heatmaps. SPSS 22.0 (IBM, Armonk, NY, USA) was employed for all statistical analyses. $P<0.05$ was considered significant.

\section{Results}

\section{Patients}

The diagnosis of PE was based on the diagnostic criteria set by the American Congress of Obstetricians and Gynecologists (2). The clinical characteristics of all recruited pregnant women are shown in Table 1. Twelve women diagnosed as having PE during the third trimester were enrolled in the PE group. Eight normotensive healthy pregnant women were in the control group. There were significant differences in the antepartum body mass index (BMI), SBP, and DBP between the PE group and control group $(P<0.05)$ (Table 1$)$, whereby the PE group had 
Table 1. Clinical information of subjects.

\begin{tabular}{lccc}
\hline & PE group $(n=12)$ & Control group $(n=8)$ & $P$ \\
\hline Age (years) & $29.0 \pm 4.2$ & $25.6 \pm 4.7$ & 0.1 II \\
Gestational weeks & $35.2 \pm 2.0$ & $34.8 \pm 4.6$ & 0.83 I \\
Pregestational body mass index $(\mathrm{BMI})\left(\mathrm{kg} / \mathrm{m}^{2}\right)$ & $25.6 \pm 5.9$ & $19.8 \pm 3.0$ & 0.0503 \\
Antepartum BMI $\left(\mathrm{kg} / \mathrm{m}^{2}\right)$ & $30.4 \pm 4.9$ & $24.6 \pm 3.3$ & $\mathbf{0 . 0 0 5 *}$ \\
Systolic blood pressure $(\mathrm{mmHg})$ & $152.8 \pm 13.4$ & $121.8 \pm 8.6$ & $<0.001 * *$ \\
Diastolic blood pressure $(\mathrm{mmHg})$ & $100.5 \pm 10.0$ & $72.3 \pm 6.0$ & $<0.001 * *$ \\
\hline
\end{tabular}

Data are expressed as the mean \pm SD. The statistically significant difference between the PE group and control group are $P<0.05(* P<0.05$; **P $<0.01)$.

higher antepartum weight and blood pressure. There were no significant differences in age, gestational age, or progestational BMI between the two groups $(P>0.05)$.

\section{Bacterial diversity in GM}

To analyze the differences of GM between the two groups, 961,060 tags from 20 stool samples were obtained (average of $48,053 \pm 1,558$ tags per sample). All tags were clustered into 1,227 OTUs.

The results of $\beta$-diversity based on PLS-DA are shown in Fig. 1. Differences in GM communities between the two groups were documented. The distribution of samples from the PE group was more concentrated, whereas the distance between samples in the control group was longer. Hence, differences in the individual composition of GM were larger in the control group, and the similarities were smaller.

We investigated the $\alpha$-diversity of GM in the two groups as described by Su and colleagues (19), which represented the mean species diversity in the overall gut. We found no significant differences in GM of the two groups. In general, the Shannon Index is used to quantify the uncertainty, whereas the Simpson Index is used to measure the degree of concentration, when individuals are classified into different types (20). However, there were no significant differences between the PE group and control group in terms of these two indices $(P>0.05)$ (Fig. 2a, b), although the Shannon Index decreased in the PE group. The Chao Index and Sobs Index also showed no significant differences $(P>0.05)$ (Fig. 2c, d), but the Chao Index and Sobs Index decreased moderately in the PE group. Meanwhile, the Observed Species Index of the control group was moderately higher than that of the PE group, although the difference was not significant $(P>0.05)$ (Fig. 2e). Taken together, these results showed that the species diversity in the PE group decreased, but there was higher distribution uniformity of bacteria in the PE group.

\section{Specific differences in GM}

We wished to further identify the differences in GM between the two groups. Hence, we analyzed the number

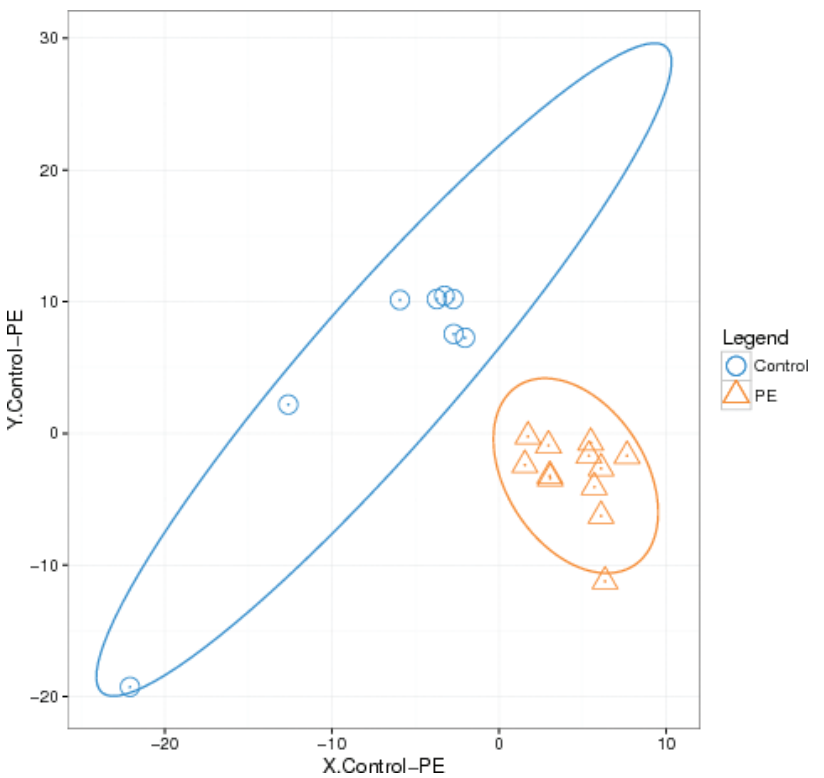

Fig. 1. The $\beta$-diversity of the microbial communities in the two groups.

and relative abundance of all microbiota at phylum, family, and genus levels from all samples.

At the phylum level, the most abundant GM in the two groups were bacteria of the phylum Firmicutes (70.22-80.91\%), Bacteroidetes (8.66-9.97\%), Actinobacteria (5.56-14.86\%), and Proteobacteria (2.8-4.6\%) (Fig. 3a, Supplementary Table 1). The relative abundance of bacteria of the phylum Firmicutes in the PE group increased slightly compared with that in the control group, although the difference was not significant $(P=0.068)$ (Fig. 3b). The relative abundance of bacteria of the phylum Bacteroidetes was not significantly different between the two groups, although there was a decreasing trend in the PE group $(P=0.73)$ (Fig. 3b). In addition, the abundance of bacteria of the phylum Proteobacteria showed a slight increase but the difference was not significant $(P=0.77)$ (Fig. 3b). However, the relative abundance of bacteria of the phylum 
a

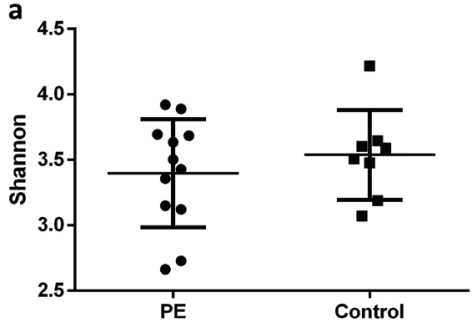

d

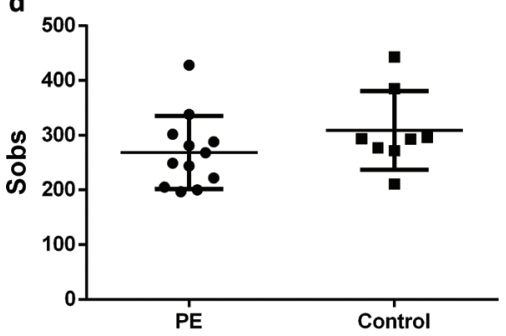

b

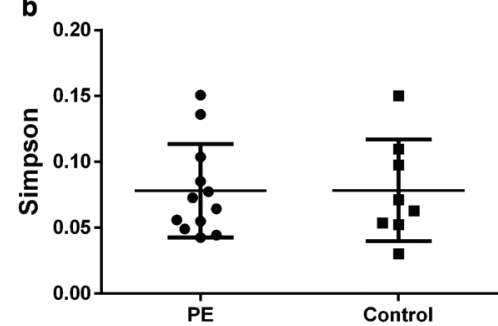

e

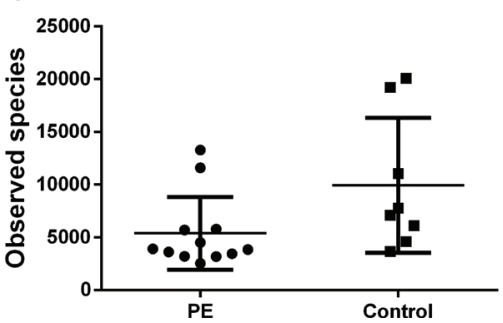

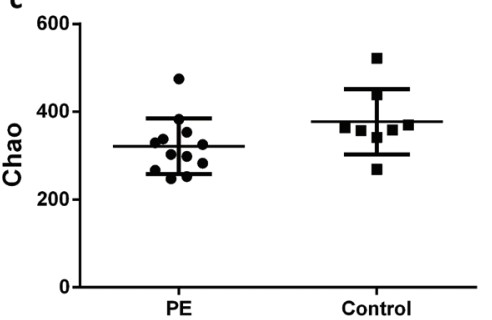

Fig. 2. The altered biodiversity of gut microbiota in the PE group compared with control group. The groups of PE $(n=12)$ and control $(n=8)$ were described in the Methods $(\mathrm{a}, \mathrm{b})$. The ecological diversity of gut microbiota in the PE and control groups was measured by Shannon Index and Simpson Index (c, d). The alpha-diversity, richness of gut microbiota, was determined by Chao Index, Sobs Index, and Observed Species Index.

Actinobacteria was decreased significantly in comparison with that of the control group $(P=0.042)$ (Fig. 3b).

The main families of bacteria in the stool samples were Lachnospiraceae, Erysipelotrichaceae, Bifidobacteriaceae, Ruminococcaceae, Bacteroidaceae, Streptococcaceae, Enterobacteriaceae, Veillonellaceae, Prevotellaceae, and Coriobacteriaceae (Fig. 4a, Supplementary Table 2). The relative abundance of bacteria of the family Bifidobacteriaceae was $3.75 \%$ in the PE group and $12.76 \%$ in the control group, respectively, and this reduction was significant in the PE group $(P=0.039)$ (Fig. 4b). The relative abundance of bacteria of the families Ruminococcaceae, Prevotellaceae, and Coriobacteriaceae in the PE group decreased compared with that in the control group, but the difference was not significant $(P>0.05)$. The relative abundance of bacteria of the families Lachnospiraceae, Bacteroidaceae, Erysipelotrichaceae, Streptococcaceae, Enterobacteriaceae, and Veillonellaceae in the PE group increased slightly compared with that in the control group, but the difference between the two groups was not significant $(P>0.05)$.

The major bacterial genera in GM were Blautia, Faecalibacterium, Ruminococcus, Bacteroides, Streptococcus, Roseburia, Bifidobacterium, Prevotella, and Eubacterium (Fig. 5a, Supplementary Table 3). Importantly, the abundance of bacteria of the Blautia was increased significantly in the PE group in comparison with that in the control group, with a relative abundance of $19.13 \%$ and $9.71 \%$, respectively $(P=0.026)$ (Fig. $5 b)$. Moreover, the relative abundance of bacteria of the genus Ruminococcus was increased significantly in the PE group compared with that in the control group $(10.32 \%$ and $6.11 \%$, respectively)
$(P=0.048)$ (Fig. 5b). However, the relative abundance of bacteria of the genus Bifidobacterium demonstrated a marked reduction in the PE group in comparison with that in the control group $(P=0.038)$ (Fig. 5b). A similar downward trend was observed for bacteria of the genera Faecalibacterium, Roseburia, and Prevotella in the PE group compared with that in the control group $(P>$ 0.05 for all). The abundance of the bacteria of the genera Bacteroides, Streptococcus, and Eubacterium was also increased, but the difference between the two groups was not significant $(P>0.05)$.

\section{Dietary analyses in pregnant women}

We wished to explore the relationship between GM and the diet, so we analyzed the dietary situation in the two groups. The intake of energy, fat, and carbohydrate was all higher than the dietary reference intake (DRIs) for pregnant women in the third trimester in the two groups, whereas the intake of dietary fiber was lower (Table 2). The protein intake in the PE group was higher than the DRIs but, in the control group, the average protein intake was lower than the DRIs. Although there was no significant difference between the two groups $(P>0.05)$ in terms of the diet, an excess intake of macronutrients in the PE group was more obvious than that in the control group, but the intake of dietary fiber was lower.

\section{Correlation between clinical parameters and GM in pregnant women}

We also investigated the correlation between maternal clinical parameters and the abundance of bacteria of certain genera in GM (Fig. 6). Remarkably, there was a significant 
a

b

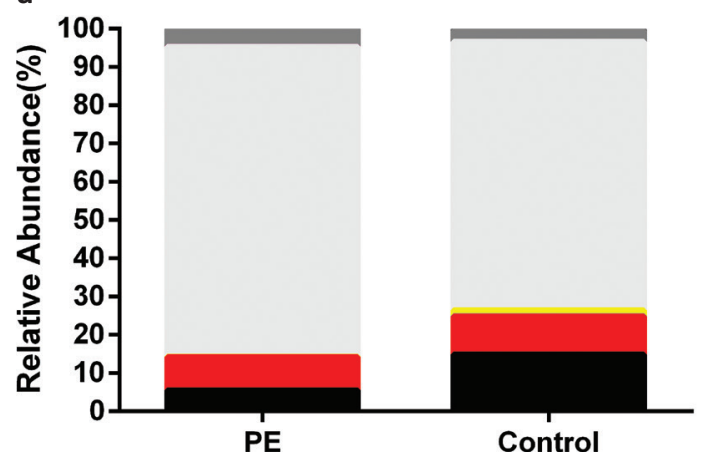

Fusobacteria

Firmicutes

Euryarchaeota

Cyanobacteria

Bacteroidetes

Actinobacteria

Proteobacteria

Synergistetes

Tenericutes

Verrucomicrobia
Firmicutes
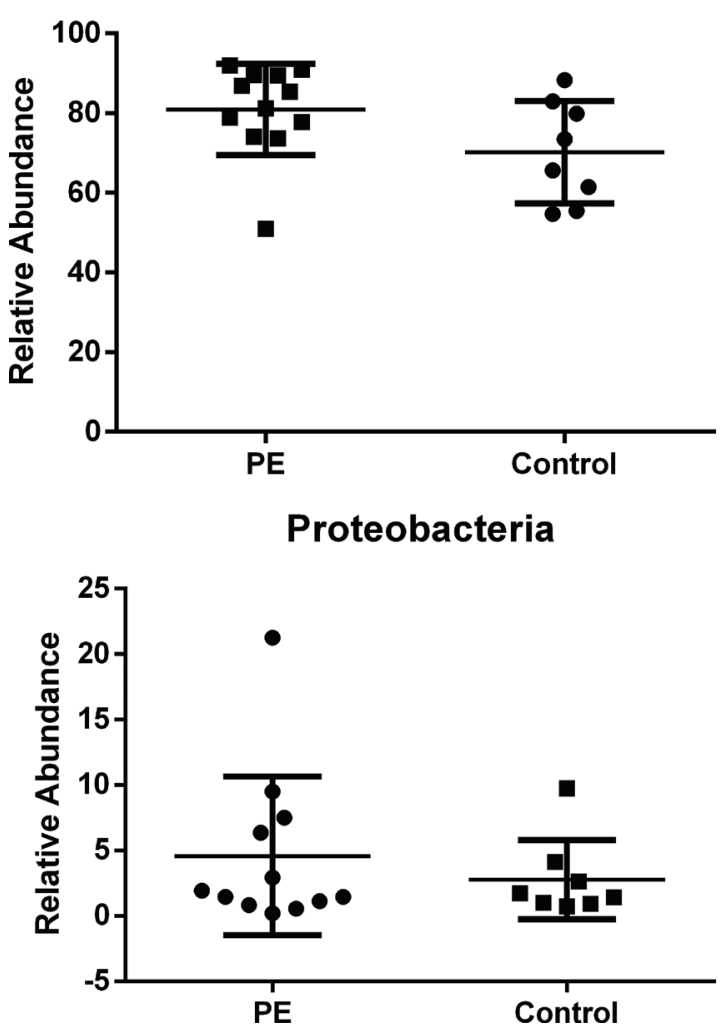

Bacteroidetes

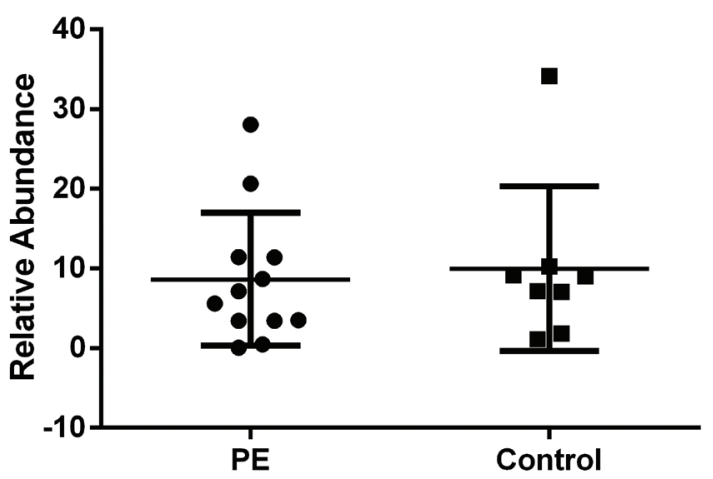

Actinobacteria

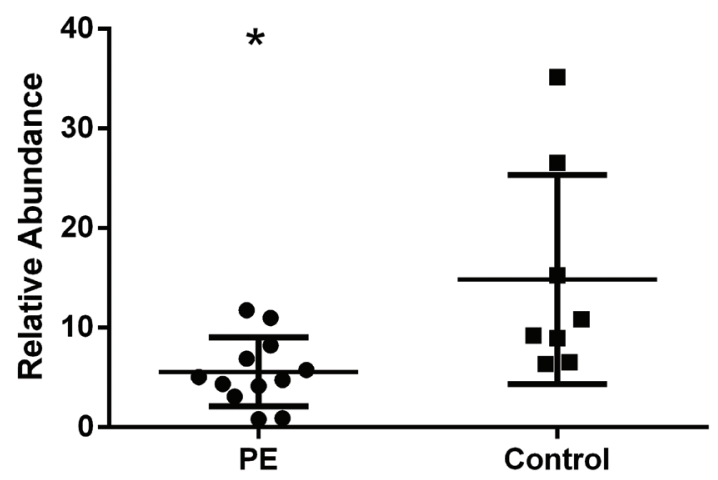

Fig. 3. The relative abundance of gut microbiota at the phylum level in the two groups. a. The relative abundance percentage of gut microbiota at the phylum level. $b$. The relative abundance comparisons of four most abundant gut microbes between the two groups $(* P=0.042)$.

positive correlation between maternal age and the relative abundance of bacteria of the genus Blautia, as well as the pregestational weight, hematocrit as well as levels of C-reactive protein and triglyceride $(P<0.05$ for all). The level of low-density lipoprotein-cholesterol was highly significantly positively correlated with the relative abundance of bacteria of the genus Blautia $(P<0.01)$. The pregestational weight, pregestational BMI, antepartum weight, antepartum BMI, and levels of lipopolysaccharide-binding protein and triglyceride were significantly positively correlated with the relative abundance of bacteria of the genus Ruminococcus ( $P<0.05$ for all). However, the relative abundance of bacteria of the genus Bifidobacterium was significantly negatively correlated with the SBP, DBP, and levels of cholesterol and aspartate aminotransferase $(P<0.05$ for all). The relative abundance of bacteria of the genus Bifidobacterium had a highly significantly negative correlation with the triglyceride level $(P<0.01)$. 
a

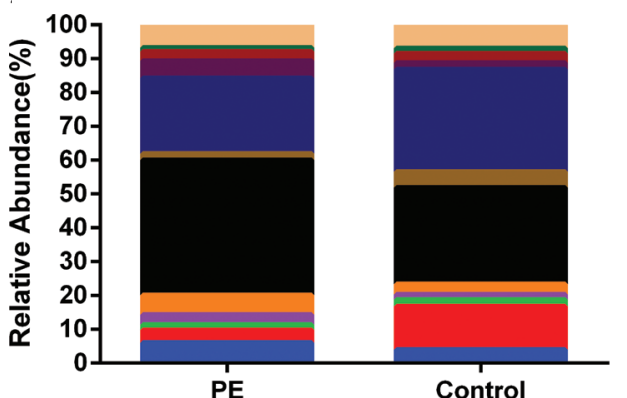

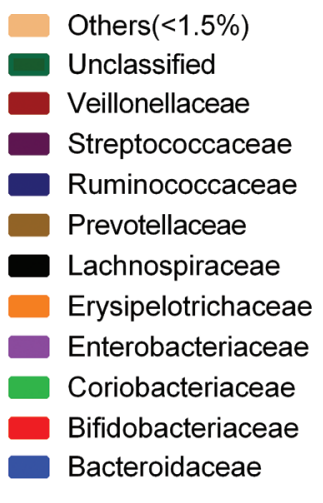

b

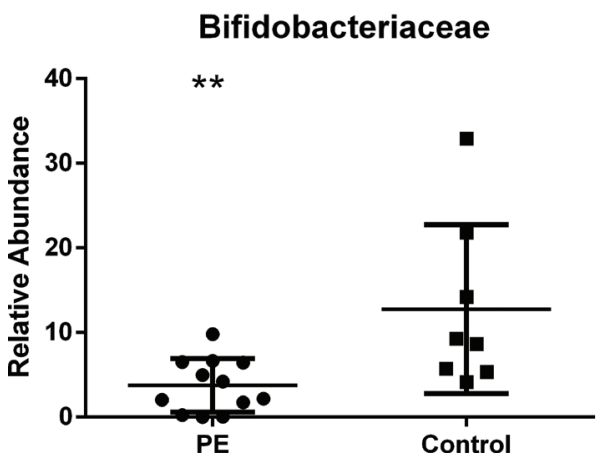

Fig. 4. The relative abundance of gut microbiota at the family level of two groups. a. The relative abundance percentage of gut microbiota at the family level. b. The relative abundance comparisons of Bifidobacteriaceae between the two groups. (* $P=0.039)$.

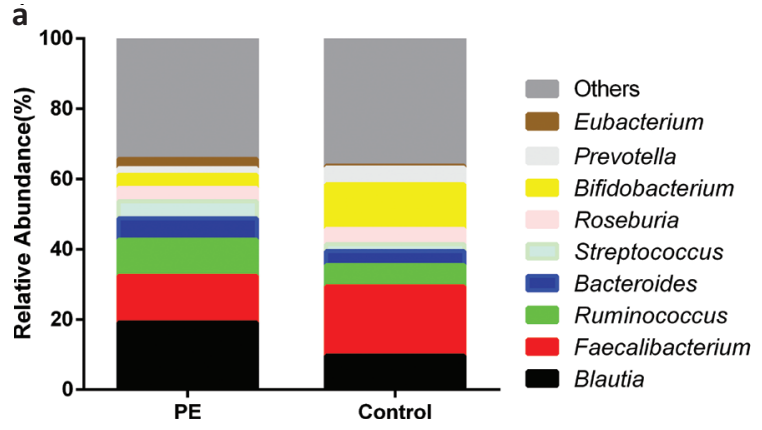

b

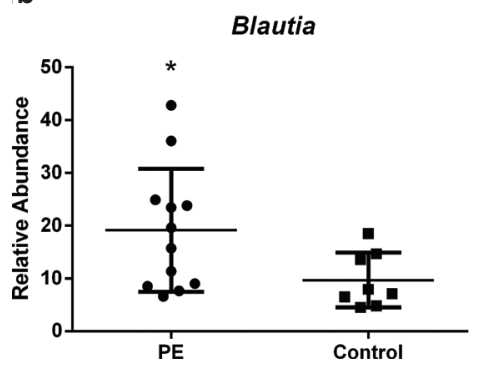

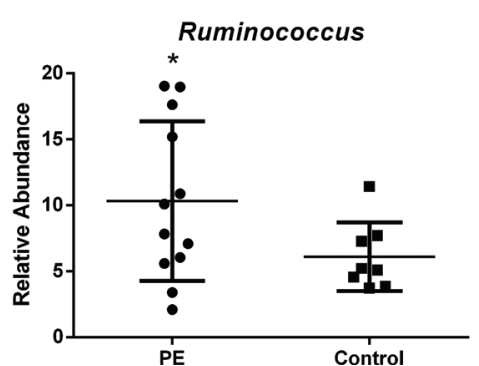

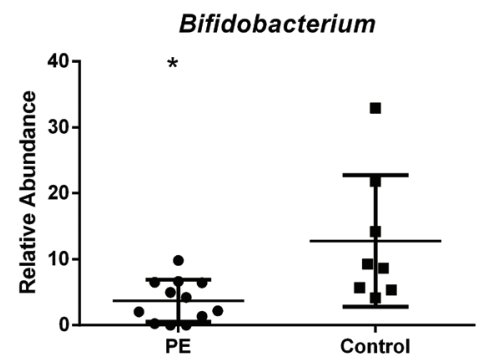

Fig. 5. The $\beta$-diversity of the microbial communities in the two groups. a. The relative abundance percentage of gut microbiota at the genus level. b. The relative abundance comparisons of three gut microbes between the two groups $(* P<0.05)$.

Table 2. Dietary situation of the two groups.

\begin{tabular}{lccccc}
\hline Group & Energy $(\mathrm{kcal})$ & Protein $(\mathrm{g})$ & Fat $(\mathrm{g})$ & Carbohydrate $(\mathrm{g})$ & Dietary fiber $(\mathrm{g})$ \\
\hline PE & $2442.75 \pm 483.59$ & $95.99 \pm 32.66$ & $89.72 \pm 32.67$ & $319.05 \pm 105.66$ & $16.07 \pm 5.84$ \\
Control & $2265.23 \pm 510.01$ & $80.90 \pm 28.70$ & $87.88 \pm 37.72$ & $294.52 \pm 72.33$ & $17.10 \pm 6.54$ \\
DRls & 2,250 & 85 & $50 \sim 75$ & $281.25 \sim 365.63$ & $25 \sim 30$ \\
$P$ & 0.524 & 0.386 & 0.924 & 0.635 & 0.637 \\
\hline
\end{tabular}

DRIs, Dietary Reference Intakes (for pregnant women in third trimester). Data are expressed as the mean \pm SD. The statistically significant difference between the PE group and control group are $P<0.05$. 


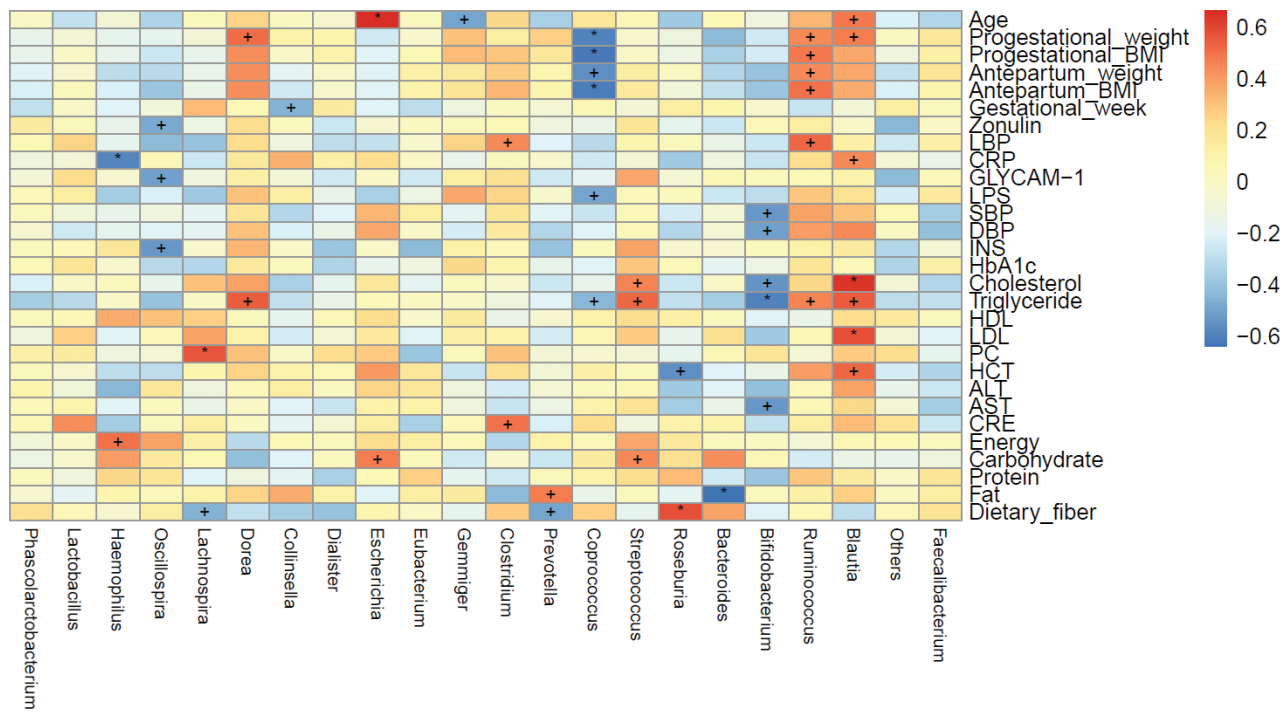

Fig. 6. The correlations between different maternal clinical parameters and gut microbiota genus in the two groups. Spearman's rank correlation coefficients and $P$-values for the correlations are shown ('+': $P<0.05$; '*': $P<0.01$ ). LBP: lipopolysaccharide-binding protein; CRP: C-reactive protein; GlyCAM-1: glycosylation cellular adhesion molecules; LPS: lipopolysaccharide; SBP: systolic blood pressure; DBP: diastolic blood pressure; INS: insulin; HbA1C: glycosylated haemoglobin; HDL: high-density lipoprotein; LDL: low-density lipoprotein; PC: platelet count; HCT: hematokrit; ALT: alanine transaminase; AST: aspartate transaminase; CRE: creatinine.

\section{Prediction of function of GM using the KEGG database}

The functional prediction of GM using the KEGG database in the two groups was analyzed by PICRUSt (17) according to OTU profiles, and 270 functional modules from the KEGG database were obtained. Thirty-one functional modules $(11.5 \%)$ were significantly different between the PE group and control group $(P<0.05)$ (Supplementary Table 5): 23 functional modules were reduced and eight functional modules were enriched in PE patients. The reduced functional modules included 'PPAR signaling pathway', 'protein metabolism', 'RNA degradation', 'VB6 metabolism', and 'adipocytokine signaling pathway'. The enriched functional modules included 'lipid metabolism', 'glycosyltransferases', 'biotin metabolism', and 'oxidative phosphorylation'. The functionally enriched genera Blautia and Ruminococcus in the PE group were significantly positively associated with all other enriched functional modules in the PE group, which showed their dominating functions in GM in the PE group (Fig. 7). The functionally reduced genus Bifidobacterium in the PE group was significantly negatively correlated with glucose metabolism, lipid metabolism, and oxidative phosphorylation, but significantly positively related with the digestion and absorption of protein.

\section{Discussion}

We sequenced 16S rRNA of stool samples from 20 pregnant women. According to the testing of species diversity and OTUs, we demonstrated that there were differences in GM between healthy pregnant women and women with PE. The Observed Species Index was lower in the PE group than that of the control group (Figs. 1, 2), which suggested that there were some dominant bacteria and a lower uniformity of bacteria distribution in the PE group compared with that in the control group. Moreover, at phylum, family, and genus levels, the amount and relative abundance of GM from the PE group displayed substantial differences to those from the control group.

Endothelial dysfunction and subsequent activation of the coagulation cascade are very important in PE pathogenesis (2). There is growing evidence suggesting that an increased inflammatory response and an imbalance of immune regulation have vital roles in early-onset PE and severe PE $(2,3)$. Placenta dysfunction is also an important pathologic basis for PE (2). Amarasekara and colleagues demonstrated bacteria in the placental tissues of women with $\mathrm{PE}$ and supported the notion that these bacteria were one of the causes of PE (21).

Liu and colleagues revealed that, in women with PE, bacteria of the phylum Cyanobacteria had an increased abundance (1.07\%) (8). We found a small decrease in the abundance of bacteria of the phylum Cyanobacteria in the PE group compared with that in the control group. However, we found that the control group had an increased abundance of bacteria of the phylum Tenericutes, a result that is consistent with that of the study from Liu and colleagues. Otherwise, the abundance of bacteria of the phylum Verrucomicrobia increased in the 


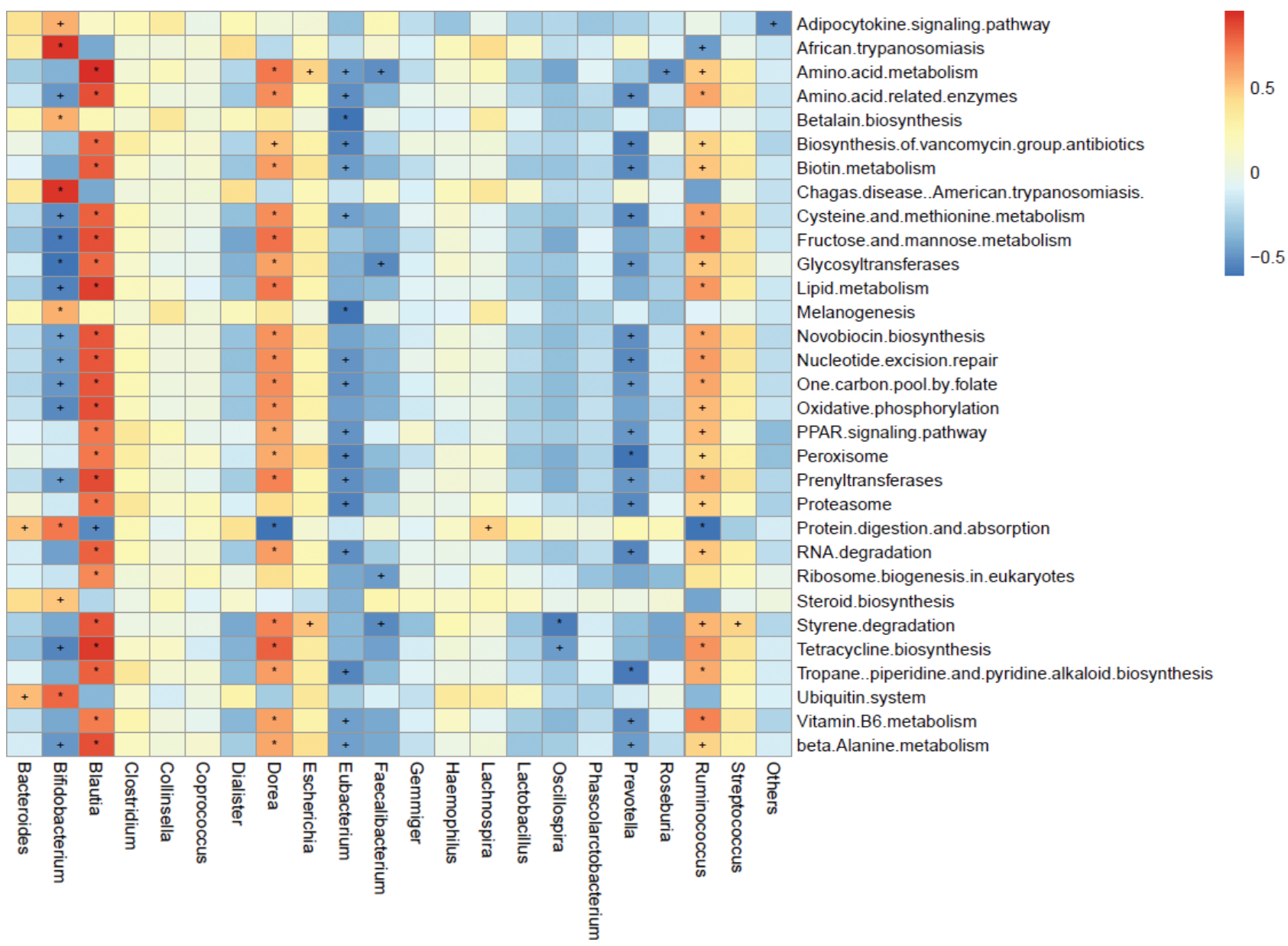

Fig. 7. The correlations between different functional modules and gut microbiota genus. Spearman's rank correlation coefficients and $P$-values for the correlations are shown ('+': $P<0.05$; '*': $P<0.01)$.

control group (8). Importantly, in our study, the relative abundance of bacteria of the phylum Actinobacteria was decreased significantly in the PE group. The abundance of bacteria from the family Bifidobacteriaceae and genus Bifidobacterium decreased significantly $(P<0.05)$ in the PE group. In general, the differences between our study and the study by Liu and colleagues may have been due to the influence of different geographic locations and dietary habits on GM. Nevertheless, the dominant different GM in women with PE reported by Liu and colleagues were similar with those reported by us.

Over the last decade, an increasing number of investigations have focused on the potential mechanisms by which intestinal microflora participate in the development of obesity (22), type-2 diabetes mellitus (23), hypertension (24), autism (25), and Parkinson's disease (26). During pregnancy, intestinal microorganisms interact with the immune system and affect the maturation of the nervous system and hormone regulation (27). In GM, a balance in the abundance of bacteria from four phyla (Bacteroidetes,
Firmicutes, Actinomycetes, and Proteobacteria) is crucial for the maintenance of intestinal immune homeostasis (28). In the intestines of healthy people, bacteria of the phyla Firmicutes and Bacteroidetes account for $>90 \%$ of flora composition, and the ratio of their abundance plays a critical part in health (29). We found that bacteria of the phylum Firmicutes were the most prominent in GM, with $>50 \%$ of the total abundance in the PE group and control group. Compared with that in the control group, the relative abundance of bacteria of the phylum Firmicutes in the PE group was increased slightly, whereas the abundance of bacteria of the phylum Bacteroidetes was essentially unchanged in the two groups. Therefore, the ratio of Firmicutes: Bacteroidetes was increased; alteration of this ratio may be one of the causes of PE. Moreover, the Firmicutes: Bacteroidetes ratio has been shown to be increased in a rat model of spontaneous hypertension and in people with systolic hypertension, data that are consistent with our findings in PE patients (29). In addition, the relative abundance of bacteria of the phylum Actinobacteria 
was decreased significantly in the PE group compared with that in the control group, whereas the abundance of bacteria of the family Bifidobacteriaceae and genus Bifidobacterium was also decreased significantly.

Studies have revealed that GM dysbiosis can contribute to hypertension and its complications (29), possibly by upregulating the inflammatory response. Levels of short-chain fatty acid (SCFAs) such as acetic acid, propionic acid, and butyric acid produced by GM are correlated with hypertension and influence vascular tone (30). GM of hypertensive rats have been shown to have fewer SCFA-producing bacteria compared with those of normotensive rats (30) and that a supply of probiotics reduces blood pressure (31). Hence, GM have important roles in the regulation of blood pressure. SCFA-producing bacteria can influence blood pressure by eliciting direct effects on vasodilation or through plasminogen activator inhibitor-1 (29). Bacteria of the genus Bifidobacterium are used commonly in probiotics and produce high levels of SCFAs, which can inhibit pathogen accumulation by reducing intestinal $\mathrm{pH}(32,33)$. Similarly, our results showed that decreased abundance of bacteria of the genus Bifidobacterium was significantly negatively related to SBP, DBP, hyperlipidemia, and the level of aspartate aminotransferase, which may be causes of PE. We also showed that the abundance of bacteria of the genus Blautia was increased significantly (almost twofold) in the PE group compared with that in the control group and that its abundance was significantly positively correlated with age, pregestational weight, and hyperlipidemia. These results are similar with those of Lv and colleagues, who reported the abundance of bacteria of the genus Blautia to be enriched significantly in women with PE (34). Pregnancy at an older age, pregestational obesity, and abnormal lipid metabolism are risk factors for PE, having bacteria of the genus Blautia is positively correlated with a higher BMI (35) and consuming a high-fat diet can result in hyperlipidemia (36). Moreover, having bacteria of the genus Blautia is positively correlated with glucose intolerance (37) and is enriched in pregnant women who were overweight, obese, or who have excessive gestational weight gain (38). Crusell and colleagues found that gestational diabetes mellitus was related to an increased abundance of bacteria of the genus Blautia, which showed that enriched abundance of bacteria of the genus Blautia is associated with an unfavorable metabolic profile (39). The abundance of bacteria of the genus Ruminococcus has been reported to be enriched in women with gestational diabetes mellitus (39) or type-2 diabetes mellitus (40). It has been demonstrated that higher abundance of bacteria of the family Ruminococcaceae (which includes the genus Ruminococcus) is positively correlated with adverse metabolic situations (41) and leptin level (an adipocyte-derived hormone that is higher in PE patients) (41-43). In general, we found that decreased abundance of bacteria of the genus Bifidobacterium was negatively correlated with SBP and DBP and involved glucose metabolism, lipid metabolism, and the oxidative-phosphorylation pathway. However, an increased abundance of bacteria of the genera Blautia and Ruminococcus was positively correlated with obesity and dyslipidemia, which involved lipid metabolism, glycosyltransferases, biotin metabolism, and the oxidative-phosphorylation pathway. Therefore, we hypothesized that the alterations mentioned above in the abundance of bacteria of the genera Bifidobacterium, Blautia, and Ruminococcus might correlate with PE progression.

It is not uncommon for pregnant women to increase food consumption to meet the growth needs of the fetus, who are thereby prone to overnutrition. According to our dietary survey, PE patients had a higher intake of carbohydrate and fat, along with lower consumption of dietary fiber, compared with those of the control group. These actions were likely to cause disorders in glucose metabolism and lipid metabolism. Lower intake of dietary fiber would supply fewer prebiotics that are beneficial for probiotics, such as bacteria of the genus Bifidobacterium. Therefore, it may be useful to increase the intake of dietary fiber up to the DRIs (but not in excess) or take more probiotics, such as functional oligosaccharides $(44,45)$. Supplementation with bacteria of the genus Bifidobacterium may also be helpful for preventing PE. Our findings support the opinions of other scholars (46-49), but clinical trials are needed to confirm our findings.

\section{Conclusions}

We reported, for the first time, GM changes in women with PE from East China. Our results indicated the potential for GM dysbiosis in PE patients and that they could be prone to suffer from metabolic syndrome. We speculate that alterations in the abundance of bacteria of certain genera (e.g. increased abundance of Blautia and Ruminococcus and decreased abundance of Bifidobacterium) were associated with PE development to some degree. Therefore, our data could help to monitor the health of pregnant women and may be helpful for preventing and assisting treatment of PE.

\section{Conflict of interest and funding}

The authors declare no conflicts of interest. The authors have not received any funding or benefits from industry or elsewhere to conduct this study.

\section{Acknowledgments}

This work was supported by Jiangsu Provincial Medical Youth Talent (QNRC2016302), Major Project of Changzhou Commission of Health (ZD201922), and Talent Project of Provincial Science and Technology Department (BRA2019160). We thank all the volunteers and all staff 
in Changzhou Maternity and Child Health Care Hospital, especially the research doctors for their excellent work.

\section{References}

1. Mol BWJ, Roberts CT, Thangaratinam S, Magee LA, Groot CJMD, Hofmeyr GJ. Pre-eclampsia. Lancet 2016; 387(10022): 999-1011. doi: 10.1016/S0140-6736(15)00070-7

2. Phipps EA, Thadhani R, Benzing T, Karumanchi SA. Preeclampsia: pathogenesis, novel diagnostics and therapies. Nat Rev Nephrol 2019; 15(5): 275-89. doi: 10.1038/s41581-019-0119-6

3. Lu HQ, Hu R. The role of immunity in the pathogenesis and development of pre- eclampsia. Scand J Immunol 2019; 90(5): e12756. doi: 10.1111/sji.12756

4. Karumanchi SA. Angiogenic factors in preeclampsia: from diagnosis to therapy. Hypertension 2016; 67(6): 1072-9. doi: 10.1161/ HYPERTENSIONAHA.116.06421

5. Eckburg PB, Bik EM, Bernstein CN, Purdom E, Dethlefsen L, Sargent M, et al. Diversity of the human intestinal microbial flora. Science 2005; 308(5728): 1635-8. doi: 10.1126/ science. 1110591

6. Round JL, Mazmanian SK. The gut microbiota shapes intestinal immune responses during health and disease. Nat Rev Immunol 2009; 9(5): 313-23. doi: 10.1038/nri2515

7. Human Microbiome Project Consortium. Structure, function and diversity of the healthy human microbiome. Nature 2012; 486(7402): 207-14. doi: 10.1038/nature 11234

8. Liu J, Yang H, Yin Z, Jiang X, Zhong H, Qiu D, et al. Remodeling of the gut microbiota and structural shifts in Preeclampsia patients in South China. Eur J Clin Microbiol Infect Dis 2017; 36(4): 713-19. doi: 10.1007/s10096-016-2853-z

9. American College of Obstetricians and Gynecologists' Committee on Practice Bulletins-Obstetrics. Gestational hypertension and preeclampsia: ACOG practice bulletin, number 222. Obstet Gynecol 2020; 135(6): e237-60. doi: 10.1097/AOG. 0000000000003891

10. Chang Y, Chen Y, Zhou Q, Wang C, Chen L, Di W, et al. Shortchain fatty acids accompanying changes in the gut microbiome contribute to the development of hypertension in patients with preeclampsia. Clin Sci (Lond) 2020; 134(2): 289-302. doi: 10.1042/CS20191253

11. Magoč T, Salzberg SL. FLASH: fast length adjustment of short reads to improve genome assemblies. Bioinformatics 2011; 27(21): 2957-63. doi: 10.1093/bioinformatics/btr507

12. Edgar RC. UPARSE: highly accurate OTU sequences from microbial amplicon reads. Nat Methods 2013; 10(10): 996-8. doi: 10.1038/nmeth.2604.

13. Edgar RC, Haas BJ, Clemente JC, Quince C, Knight R. UCHIME improves sensitivity and speed of chimera detection. Bioinformatics 2011; 27(16): 2194-200. doi: 10.1093/bioinformatics/ btr381

14. Caporaso JG, Kuczynski J, Stombaugh J, Bittinger K, Bushman FD, Costello EK, et al. QIIME allows analysis of high-throughput community sequencing data. Nat Methods 2010; 7(5): 335-6. doi: 10.1038/nmeth.f.303

15. Edgar RC. Search and clustering orders of magnitude faster than BLAST. Bioinformatics 2010; 26(19): 2460-1. doi: 10.1093/ bioinformatics/btq461

16. James JL, Whitley GS, Cartwright JE. Pre-eclampsia: fitting together the placental, immune and cardiovascular pieces. J Pathol 2010; 221(4): 363-78. doi: 10.1002/path.2719

17. Langille MG, Zaneveld J, Caporaso JG, McDonald D, Knights D, Reyes JA, et al. Predictive functional profiling of microbial communities using 16S rRNA marker gene sequences. Nat Biotechnol 2013; 31(9): 814-21. doi: 10.1038/nbt.2676

18. Kanehisa M, Furumichi M, Tanabe M, Sato Y, Morishima K. KEGG: new perspectives on genomes, pathways, diseases and drugs. Nucleic Acids Res 2017; 45(D1): D353-61. doi: 10.1093/ nar/gkw1092

19. Su M, Nie Y, Shao R, Duan S, Jiang Y, Wang M, et al. Diversified gut microbiota in newborns of mothers with gestational diabetes mellitus. PLoS One 2018; 13(10): e0205695. doi: 10.1371/ journal.pone. 0205695

20. Luo M, Liu Y, Wu P, Luo DX, Sun Q, Zheng H, et al. Alternation of gut microbiota in patients with pulmonary tuberculosis. Front Physiol 2017; 8: 822. doi: 10.3389/fphys.2017.00822

21. Amarasekara R, Jayasekara RW, Senanayake H, Dissanayake $\mathrm{VH}$. Microbiome of the placenta in pre-eclampsia supports the role of bacteria in the multifactorial cause of pre-eclampsia. $\mathrm{J}$ Obstet Gynaecol Res 2015; 41(5): 662-9. doi: 10.1111/jog.12619

22. Qin J, Li Y, Cai Z, Li S, Zhu J, Zhang F, et al. A metagenome-wide association study of gut microbiota in type 2 diabetes. Nature 2012; 490(7418): 55-60. doi: 10.1038/nature11450

23. Verdam FJ, Fuentes S, de Jonge C, Zoetendal EG, Erbil R, Greve JW, et al. Human intestinal microbiota composition is associated with local and systemic inflammation in obesity. Obesity (Silver Spring) 2013; 21(12): E607-15. doi: 10.1002/oby.20466

24. Yang T, Santisteban MM, Rodriguez V, Li E, Ahmari N, Carvajal JM, et al. Gut dysbiosis is linked to hypertension. Hypertension 2015; 65(6): 1331-40. doi: 10.1161/HYPERTENSIONAHA.115.05315

25. Strati F, Cavalieri D, Albanese D, De Felice C, Donati C, Hayek J, et al. New evidences on the altered gut microbiota in autism spectrum disorders. Microbiome 2017; 5(1): 24. doi: 10.1186/s40168-017-0242-1

26. Li W, Wu X, Hu X, Wang T, Liang S, Duan Y, et al. Structural changes of gut microbiota in Parkinson's disease and its correlation with clinical features. Sci China Life Sci 2017; 60(11): 1223-33. doi: 10.1007/s11427-016-9001-4

27. Koren O, Goodrich JK, Cullender TC, Spor A, Laitinen K, Bäckhed HK, et al. Host remodeling of the gut microbiome and metabolic changes during pregnancy. Cell 2012; 150(3): 470-80. doi: 10.1016/j.cell.2012.07.008

28. Mariat D, Firmesse O, Levenez F, Guimarăes V, Sokol H, Doré J, et al. The Firmicutes/Bacteroidetes ratio of the human microbiota changes with age. BMC Microbiol 2009; 9: 123. doi: 10.1186/1471-2180-9-123

29. Gomez-Arango LF, Barrett HL, McIntyre HD, Callaway LK, Morrison M, Dekker Nitert M, et al. Increased systolic and diastolic blood pressure is associated with altered gut microbiota composition and butyrate production in early pregnancy. Hypertension 2016; 68(4): 974-81. doi: 10.1161/ HYPERTENSIONAHA.116.07910

30. Mell B, Jala VR, Mathew AV, Byun J, Waghulde H, Zhang Y, et al. Evidence for a link between gut microbiota and hypertension in the Dahl rat. Physiol Genomics 2015; 47(6): 187-97. doi: 10.1152/physiolgenomics.00136.2014

31. Gómez-Guzmán $\mathrm{M}$, Toral $\mathrm{M}$, Romero $\mathrm{M}$, Jiménez $\mathrm{R}$, Galindo P, Sánchez M, et al. Antihypertensive effects of probiotics Lactobacillus strains in spontaneously hypertensive rats. Mol Nutr Food Res 2015; 59(11): 2326-36. doi: 10.1002/ mnfr. 201500290

32. Roberfroid M, Gibson GR, Hoyles L, McCartney AL, Rastall R, Rowland I, et al. Prebiotic effects: metabolic and health benefits. Br J Nutr 2010; 104 Suppl 2: S1-63. doi: 10.1017/ S0007114510003363 
33. Park HE, Kim YJ, Do KH, Kim JK, Ham JS, Lee WK. Effects of Queso blanco cheese containing Bifidobacterium longum KACC 91563 on the intestinal microbiota and short chain fatty acid in healthy companion dogs. Korean J Food Sci Anim Resour 2018; 38(6): 1261-72. doi: 10.5851/kosfa.2018.e62

34. Lv LJ, Li SH, Li SC, Zhong ZC, Duan HL, Tian C, et al. Early-onset preeclampsia is associated with gut microbial alterations in antepartum and postpartum women. Front Cell Infect Microbiol 2019; 9: 224. doi: 10.3389/fcimb.2019.00224

35. Ottosson F, Brunkwall L, Ericson U, Nilsson PM, Almgren P, Fernandez C, et al. Connection between BMI-related plasma metabolite profile and gut microbiota. J Clin Endocrinol Metab 2018; 103(4): 1491-501. doi: 10.1210/jc.2017-02114. 224

36. Kashtanova DA, Tkacheva ON, Doudinskaya EN, Strazhesko ID, Kotovskaya YV, Popenko AS, et al. Gut microbiota in patients with different metabolic statuses: Moscow study. Microorganisms 2018; 6(4): 98. doi: 10.3390/microorganisms6040098

37. Egshatyan L, Kashtanova D, Popenko A, Tkacheva O, Tyakht A, Alexeev D, et al. Gut microbiota and diet in patients with different glucose tolerance. Endocr Connect 2016; 5(1): 1-9. doi: 10.1530/EC-15-0094

38. Stanislawski MA, Dabelea D, Wagner BD, Sontag MK, Lozupone CA, Eggesbø M. Pre-pregnancy weight, gestational weight gain, and the gut microbiota of mothers and their infants. Microbiome 2017; 5(1): 113. doi: 10.1186/s40168-017-0332-0

39. Crusell MKW, Hansen TH, Nielsen T, Allin KH, Rühlemann MC, Damm P, et al. Gestational diabetes is associated with change in the gut microbiota composition in third trimester of pregnancy and postpartum. Microbiome 2018; 6(1): 89 . doi: 10.1186/s40168-018-0472-x

40. Zhang X, Shen D, Fang Z, Jie Z, Qiu X, Zhang C, et al. Human gut microbiota changes reveal the progression of glucose intolerance. PLoS One 2013; 8(8): e71108. doi: 10.1371/journal.pone.0071108

41. Gomez-Arango LF, Barrett HL, McIntyre HD, Callaway LK, Morrison M, Dekker Nitert M, et al. Connections between the gut microbiome and metabolic hormones in early pregnancy in overweight and obese women. Diabetes 2016; 65(8): 2214-23. doi: $10.2337 / \mathrm{db} 16-0278$

42. Taylor BD, Ness RB, Olsen J, Hougaard DM, Skogstrand K, Roberts JM, et al. Serum leptin measured in early pregnancy is higher in women with preeclampsia compared with normotensive pregnant women. Hypertension 2015; 65(3): 594-9. doi: 10.1161/HYPERTENSIONAHA.114.03979
43. Miehle K, Stepan H, Fasshauer M. Leptin, adiponectin and other adipokines in gestational diabetes mellitus and pre-eclampsia. Clin Endocrinol (Oxf) 2012; 76(1): 2-11. doi: 10.1111/j.1365-2265.2011.04234.x

44. Sohn K, Underwood MA. Prenatal and postnatal administration of prebiotics and probiotics. Semin Fetal Neonatal Med 2017; 22(5): 284-9. doi: 10.1016/j.siny.2017.07.002

45. VandeVusse L, Hanson L, Safdar N. Perinatal outcomes of prenatal probiotic and prebiotic administration: an integrative review. J Perinat Neonatal Nurs 2013; 27(4): 288-301; quiz E1-2. doi: 10.1097/JPN.0b013e3182a1e15d

46. Brantsaeter AL, Myhre R, Haugen M, Myking S, Sengpiel V, Magnus $\mathrm{P}$, et al. Intake of probiotic food and risk of preeclampsia in primiparous women: the Norwegian mother and child cohort study. Am J Epidemiol 2011; 174(7): 807-15. doi: 10.1093/ aje/kwr168

47. Lindsay KL, Walsh CA, Brennan L, McAuliffe FM. Probiotics in pregnancy and maternal outcomes: a systematic review. J Matern Fetal Neonatal Med 2013; 26(8): 772-8. doi: 10.3109/ 14767058

48. Nordqvist M, Jacobsson B, Brantsæter AL, Myhre R, Nilsson S, Sengpiel V. Timing of probiotic milk consumption during pregnancy and effects on the incidence of preeclampsia and preterm delivery: a prospective observational cohort study in Norway. BMJ Open 2018; 8(1): e018021. doi: 10.1136/ bmjopen-2017-018021

49. de Brito AJL, de Oliveira Y, Carvalho NNC, Cavalcante RGS, Souza ELD. Gut microbiota and probiotic intervention as a promising therapeutic for pregnant women with cardiometabolic disorders: present and future directions. Pharmacol Res 2019; 145: 104252. doi: 10.1016/j.phrs.2019.104252

\footnotetext{
*Huiyan Wang

Department of Obstetrics and Gynecology

Changzhou Maternity and Child Health Care Hospital affiliated

to Nanjing Medical University

No. 16, Dingxiang Road

Zhonglou District, Changzhou, 213003

jiangsu Province, China

E-mail: huiyanwang@njmu.edu.cn
} 